\title{
Vaccine Antigen
}

National Cancer Institute

\section{Source}

National Cancer Institute. Vaccine Antigen. NCI Thesaurus. Code C2835.

A vaccine component consisting of a protein, small peptide, or polysaccharide, natural or synthetic, that once administered is bound by antibody, thereby inducing mechanisms of antibody-dependent cell-mediated cytotoxicity (ADCC). In cancer immunotherapy, a cancer vaccine containing specific tumor-associated antigens (TAAs) may be used to boost an immune response ag ainst a tumor expressing those TAAs. ( $\mathrm{NCI04)}$ 